\title{
Entanglement Area Law in Disordered Free Fermion Anderson Model in One, Two, and Three Dimensions
}

\author{
Mohammad Pouranvari, Yuhui Zhang, and Kun Yang \\ National High Magnetic Field Laboratory and Department of Physics, Florida State University, Tallahassee, FL 32306, USA \\ Correspondence should be addressed to Mohammad Pouranvari; pouranvari@magnet.fsu.edu
}

Received 12 October 2014; Revised 11 February 2015; Accepted 12 February 2015

Academic Editor: Jan A. Jung

Copyright (C) 2015 Mohammad Pouranvari et al. This is an open access article distributed under the Creative Commons Attribution License, which permits unrestricted use, distribution, and reproduction in any medium, provided the original work is properly cited.

\begin{abstract}
We calculate numerically the entanglement entropy of free fermion ground states in one-, two-, and three-dimensional Anderson models and find that it obeys the area law as long as the linear size of the subsystem is sufficiently larger than the mean free path. This result holds in the metallic phase of the three-dimensional Anderson model, where the mean free path is finite although the localization length is infinite. Relation between the present results and earlier ones on area law violation in special one-dimensional models that support metallic phases is discussed.
\end{abstract}

\section{Introduction}

Recent years have witnessed tremendous progress in the study of entanglement in condensed matter/many-body physics. Among these studies, free fermion systems play a very special role [1]. Simple as they may seem, fermions are intrinsically nonlocal, due to the anticommutation relation fermion operators satisfy, no matter how far apart they are. Such nonlocality shows up as enhanced entanglement in the ground state; for example, for many years Fermi sea states were the only known ground states whose block entanglement entropy (EE) violates the area law satisfied by most ground states above 1D [2-4]. It is only recently shown that a similar violation occurs in interacting fermion systems in the Fermi liquid phase [5] and bosonic models with excitation spectra that vanish on (extended) Bose surfaces [6]. The existence of sharp Fermi or Bose surfaces is crucial for the area law violation in translationally invariant systems.

Comparatively much less effort has been devoted to studies of fermions in the presence of disorder potential. In a recent work [7] we studied two very special (onedimensional) 1D models that exhibit free fermion metalinsulator transition (MIT) and found area law violation in the metallic phase, despite the presence of disorder, and thus absence of sharp Fermi surface (actually points in 1D). It was conjectured [7] that as long as the system is metallic, namely, states are delocalized at the Fermi energy, there will be area law violation. In the present work we test this conjecture by performing detailed numerical studies of the Anderson model [8] in one, two, and three dimensions. We find that the area law is actually respected in all cases, including the metallic phase in 3D. We do observe an enhancement (beyond area law) as systems sizes increase while below the mean free path; such enhancement disappears once the system size becomes sufficiently bigger than the mean free path. The origin of the difference between the Anderson model studied here and the special models studied earlier [7] will be discussed.

The remainder of the paper is organized as follows. In Section 2 we introduce our model and numerical method for calculating EE. Results of our calculations are presented in Section 3. Section 4 offers a summary and discussions on our results.

\section{Model and Basic Considerations}

Anderson model in $D$ dimension is a model with constant nearest neighbor hopping term and random on-site energy $\varepsilon$ :

$$
H=\sum_{\vec{r}} \sum_{\vec{d}}\left(c_{\vec{r}}^{\dagger} c_{\vec{r}+\vec{d}}+c_{\vec{r}+\vec{d}}^{\dagger} c_{\vec{r}}\right)+\sum_{\vec{r}} \varepsilon_{\vec{r}} c_{\vec{r}}^{\dagger} c_{\vec{r}}
$$

where summation is over all sites in $D$ dimensional hyper cubic lattice (with lattice constant set to be 1 ) and $\vec{d}$ is a vector connecting a site to its nearest neighbor. $\varepsilon$ 's are uniformly 
distributed between $-w / 2$ and $w / 2$ [9]. The Fermi energy $E_{F}$ is set to be 0 (so the lattice is half-filled) in all cases, while in $2 \mathrm{D}$ we also study $E_{F}=1$ to avoid the van Hove singularity at the band center. We consider cubic-shaped finite-size systems with linear size $L$ and open-boundary conditions. We then divide them into two equal subsystems $A$ and $B$ with size $L^{D-1} \times(L / 2)$ and calculate the disorder-averaged entanglement entropy as detailed below.

For a system in a pure state $|\psi\rangle$, the density matrix is $\rho=|\psi\rangle\langle\psi|$. Reduced density matrix of each subsystem (A or $B$ ) is obtained by tracing over degrees of freedom of the other subsystem: $\rho^{A / B}=\operatorname{tr}_{B / A}(\rho)$. Block EE between the two subsystems is $\mathrm{EE}=-\operatorname{tr}\left(\rho^{A} \ln \rho^{A}\right)=-\operatorname{tr}\left(\rho^{B} \ln \rho^{B}\right)$. For a single Slater-determinant ground state,

$$
\rho^{A / B}=\frac{1}{Z} e^{-H^{A / B}}
$$

are characterized by free fermion entanglement Hamiltonians

$$
H^{A / B}=\sum_{i j} h_{i j}^{A / B} c_{i}^{\dagger} c_{j}
$$

where $Z$ is determined by the normalization condition $\operatorname{tr} \rho^{A / B}=1$. We calculate EE using the method of [10] by diagonalizing correlation matrix of subsystem $A$

$$
C_{m n}=\left\langle c_{m}^{\dagger} c_{n}\right\rangle
$$

and find its eigenvalues $\zeta$ 's. Then EE takes the form

$$
\mathrm{EE}=-\sum_{l=1}^{N_{A}}\left[\zeta_{l} \ln \left(\zeta_{l}\right)+\left(1-\zeta_{l}\right) \ln \left(1-\zeta_{l}\right)\right],
$$

where $N_{A}$ is number of sites in subsystem $A$.

In one and two dimensions, all states are localized with any finite disorder. However there is an important difference between them: in 1D the localization length $\xi$ is of the same order as mean free path $\ell$, while in $2 \mathrm{D}$ we have $\xi \gg \ell$ for weak disorder. In $3 \mathrm{D}$ there is a metal-insulator transition (MIT) at a critical value of disorder strength $w_{c} \approx 16$ [11], where $\xi$ diverges. The focus of our numerical calculation is the interplay of the three different length scales, mean free path $\ell$ (calculated perturbatively in the Appendix), localization length $\xi$, and (sub)system size $L$, and their effects on entanglement.

\section{Results}

3.1. Anderson Model in One and Two Dimensions. In these two cases, all states are localized as long as $w>0$.

Figure 1 shows $1 \mathrm{D}$ EE as a function of system size $L$ for different values of $w$. As size $L$ increases, EE grows logarithmically for $w=0$ as expected. For $w>0$, EE grows with $L$ in a manner similar to the disorder free case up to some point and then saturates, indicating area law is obeyed for sufficiently large system sizes. We find substantial deviation (from $w=0$ case) starts when the system size $L$ reaches the mean free path $\ell$, and saturation occurs around $L \approx 3 \ell$. We note in $1 \mathrm{D}$ that we have the localization length $\xi \sim \ell$; it is thus

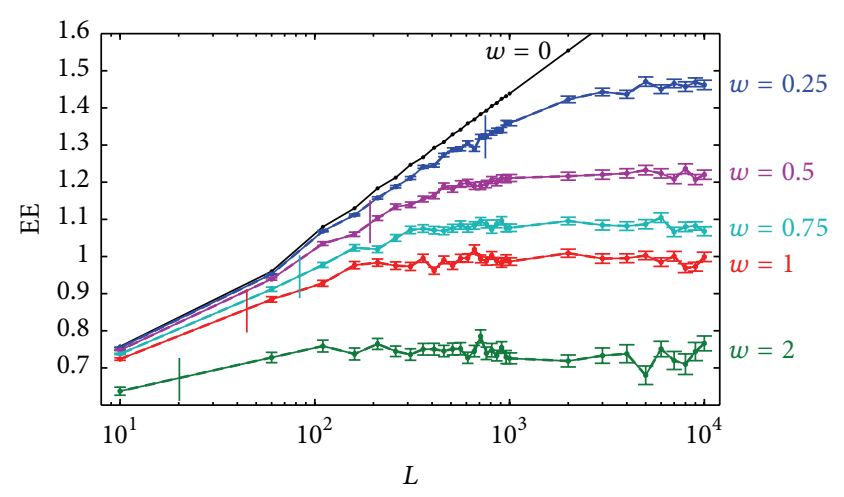

Figure 1: Entanglement entropy of one-dimensional Anderson model in log-linear scale for different value of disorder strength $w$. $E_{F}=0$. Mean free path corresponding to selected $w$ 's is indicated as a vertical line. Horizontal axis is the linear size of the system, $L$, varying from 10 up to 10000 . The number of samples at each point of $L$ is 200 . The strange break in the small $w$ 's including $w=0$ case is a consequence of small size effect in calculating average EE. We see that for bigger sizes the behavior is more smooth.

not immediately clear at this point which of the two controls is the crossover.

In Figures 2 and 3, 2D EE divided by boundary length $L$ (to account for the area law contribution) as a function of $L$ for different values of disorder strength is plotted for $E_{F}=0$ and $E_{F}=1$, respectively. We see again that, for $w=0$, there is a logarithmic growth as expected [2,3], while for $w>0$ such growth stops beyond certain length scale, indicating area law behavior. This is particularly clear in Figure 2, for $w \geq 1$; for smaller $w$ the deviation from the $w=0$ behavior and tendency toward saturation are also very obvious. In this case $\ell$ is not well defined perturbatively due to van Hove singularity, but the localization length $\xi$ is much bigger than the system sizes studied here (numerical calculations of [12] show that $\xi$ is in order of $10^{4}$ for $w=3$ and in order of $10^{6}$ for $w=2$ and exponentially bigger for smaller $w$ 's), indicating $\xi$ plays no role in the size dependence of EE.

For $E_{F}=1$ (Figure 3), we again find deviation from the $w=0$ behavior, and tendency toward saturation starts when system size $L$ reaches the mean free path $\ell$. We note in this case that we have $\xi \sim \ell e^{\pi k_{F} \ell / 2} \gg \ell$ for small $w$; for example, at $w=1$ we expect $\xi \sim 10^{36} \ell$ !. We thus again find that while $\xi$ controls the extensiveness of the fermion wave function at the Fermi level, it does not control the size dependence of EE.

3.2. Anderson Model in Three Dimensions. In three dimensions, there is a critical value of disorder strength, $w_{c} \approx 16$, where a metal-insulator transition occurs. States at the Fermi level are delocalized for $w<w_{c}$. In Figure 4, EE divided by boundary area $L^{2}$ (to account for the area law contribution) as a function of $L$ for different values of disorder strength is plotted. Similar to the $1 \mathrm{D}$ and 2D cases, we find a logarithmic growth for $w=0$, while for $w>0$ we find deviation from such growth, and tendency toward saturation starts when the system size $L$ reaches mean free path $\ell$. It is particularly worth noting that there is no obvious change of the behavior of EE near $w=w_{c} \approx 16$, which is highlighted in the figure; area law 


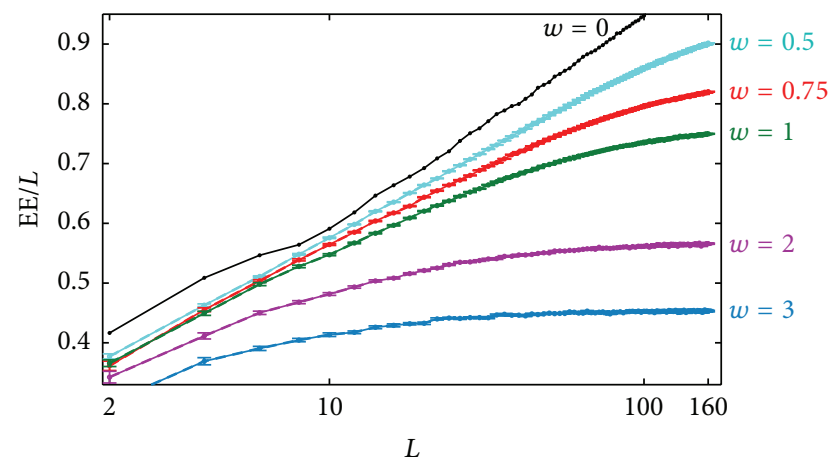

FIGURE 2: Entanglement entropy divided by linear system size of two-dimensional Anderson model in log-linear scale for different value of disorder strength $w . E_{F}=0$. Horizontal axis is the linear size of the system, $L$. The total system has $L \times L$ sites. The number of samples at each point of $L$ is 100 .

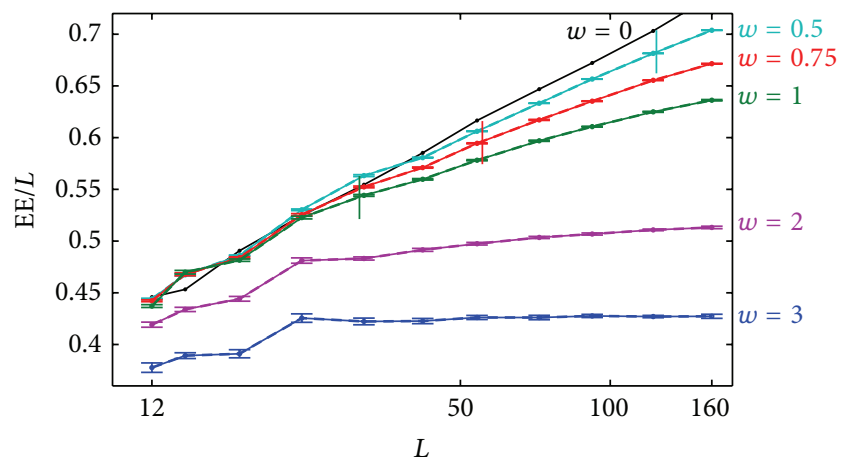

FIGURE 3: Entanglement entropy divided by linear system size of two-dimensional Anderson model in log-linear scale for different value of disorder strength $w . E_{F}=1$. Mean free path corresponding to selected $w$ 's is indicated as a vertical line. Horizontal axis is the linear size of the system, $L$. The total system has $L \times L$ sites. The number of samples at each point of $L$ is 100 .

behavior is clearly seen on both sides of $w_{c}$. We thus conclude that entanglement area law is respected in both the metallic and insulating phases of the 3D Anderson model, as long as there is finite disorder strength.

\section{Discussion and Summary}

In this work we find, through explicit numerical calculations, that entanglement entropy of free fermion systems obeys the area law in the ground states of one-, two-, and threedimensional Anderson models, for subsystems whose linear size is sufficiently bigger than the mean free path. This result holds in the metallic phase of the three-dimensional case, where states at the Fermi energy are extended. Localization length, either finite or infinite, appears to play no special role in the size dependence of entanglement entropy.

This behavior is in sharp contrast to those of two special 1D models studied earlier [7], where we found violation of area law in the metallic phase. We now discuss the source of difference in these models. The first one is the random dimer model [13], which has a very special feature that there

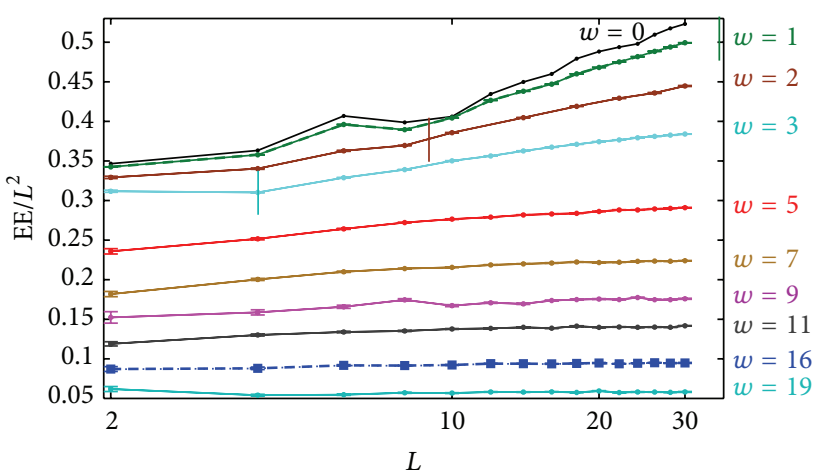

FIGURE 4: Entanglement entropy divided by square of linear system size of three-dimensional Anderson model in log-linear scale for different value of disorder strength $w . E_{F}=0$. Mean free path corresponding to selected $w^{\prime}$ 's is indicated as a vertical line. Data points corresponding to $w_{c}=16$ are indicated by blue square. Horizontal axis is the linear size of the system, $L$. The total system has $L \times L \times L$ sites. The number of samples is 100 for small sizes and 10 for large sizes.

is no back scattering at a special resonance energy, as a result of which the system is metallic when the Fermi energy matches the resonance energy. In this case the mean free path $\ell$ is infinity (and the fermion motion is ballistic instead of diffusive as in ordinary diffusive metals), resulting in the area law violation, consistent with findings of the present work.

The second special model studied in [7] involves powerlaw long-range hopping [14], and the system is metallic when the decay exponent is small enough. In this case the fermion can hop over arbitrarily large distances; thus mean free path is not well defined. More importantly, in the metallic phase fermion motion is super diffusive [14], different from that in the 3D Anderson model.

Combining earlier results with the present ones, we conclude that while violation of entanglement area law does not necessarily require a sharp Fermi surface (which is destroyed by disorder), it does not occur in diffusive metals. It can occur, however, in metallic phases of disordered free fermion models where the fermion motion is super diffusive.

While in this paper we studied noninteracting fermions, a recent paper studied the role of localization length in the case of interacting fermions [15]. Also another paper [16] proved the area law in the localized regime of the Anderson model, although our numerical calculations demonstrate area law in the metallic phase as well.

Note Added. While the present paper is being written up, a related preprint [17] appeared on the arxiv reaching a very similar conclusion. The numerics of that work are limited to quasi-1D strips.

\section{Appendix}

\section{Calculation of Mean Free Path as a Function of Random Potential Strength}

We explain the calculation of mean free path here. We assume that $\varepsilon$ 's are uncorrelated random numbers with uniform 
distribution, that is, a white noise uniform distribution. First we need to calculate the ensemble averaged transition rate using Fermi's golden rule:

$$
\frac{1}{\tau_{\vec{k}}}=\frac{2 \pi}{\hbar} \frac{L^{D}}{(2 \pi)^{D}} \int d^{D} \vec{q}\left\langle\left|\left\langle\psi_{\vec{q}+\vec{k}}\left|\varepsilon_{i}\right| \psi_{\vec{k}}\right\rangle\right|^{2}\right\rangle \delta\left(E_{\vec{k}+\vec{q}}-E_{\vec{k}}\right),
$$

in which \langle\rangle stands for ensemble average and $D$ is the dimension. We calculate the mean free path at the Fermi level, which is chosen to be $E_{F}=0$ for $1 \mathrm{D}$ and $3 \mathrm{D}$ and $E_{F}=1$ for 2D. Also we know

$$
\left\langle\left|\left\langle\psi_{\vec{q}+\vec{k}}\left|\varepsilon_{i}\right| \psi_{\vec{q}}\right\rangle\right|^{2}\right\rangle=\sum_{\vec{r}} \sum_{\vec{r}^{\prime}} \frac{e^{-i \vec{q} \cdot \vec{r}}}{L^{D}} \frac{e^{+i \vec{q} \cdot \vec{r}^{\prime}}}{L^{D}}\left\langle\varepsilon_{\vec{r}} \varepsilon_{\vec{r}^{\prime}}\right\rangle,
$$

where for a uniform distribution we have

$$
\left\langle\varepsilon_{\vec{r}} \varepsilon_{\vec{r}^{\prime}}\right\rangle=\frac{w^{2}}{12} \delta_{\vec{r}, \vec{r}^{\prime}}
$$

Thus, finally we have

$$
\frac{1}{\tau}=\frac{2 \pi}{\hbar} \frac{1}{(2 \pi)^{D}} \frac{w^{2}}{12} \int d^{D} \vec{q} \delta\left(E_{\vec{q}}-E_{F}\right) .
$$

Then, $\ell=v \tau$, where $v$ is the average velocity of electron on Fermi surface. Calculated mean free path in 1, 2, and 3 dimensions at specific Fermi energy, $E_{F}$, is

$$
\ell \approx \begin{cases}\frac{48}{w^{2}} & E_{F}=0, D=1 \\ \frac{31}{w^{2}} & E_{F}=1, D=2 \\ \frac{36}{w^{2}} & E_{F}=0, D=3 .\end{cases}
$$

\section{Conflict of Interests}

The authors declare that there is no conflict of interests regarding the publication of this paper.

\section{Acknowledgment}

This research is supported by DOE Grant no. DE-SC0002140.

\section{References}

[1] I. Peschel and V. Eisler, "Reduced density matrices and entanglement entropy in free lattice models," Journal of Physics A. Mathematical and Theoretical, vol. 42, no. 50, Article ID 504003, 30 pages, 2009.

[2] D. Gioev and I. Klich, "Entanglement entropy of fermions in any dimension and the widom conjecture," Physical Review Letters, vol. 96, Article ID 100503, 2006.

[3] M. M. Wolf, "Violation of the entropic area law for fermions," Physical Review Letters, vol. 96, Article ID 010404, 2006.

[4] B. Swingle, "Entanglement entropy and the Fermi surface," Physical Review Letters, vol. 105, no. 5, Article ID 050502, 2010.
[5] W. Ding, A. Seidel, and K. Yang, "Entanglement entropy of fermi liquids via multidimensional bosonization," Physical Review X, vol. 2, no. 1, Article ID 011012, 18 pages, 2012.

[6] H.-H. Lai, K. Yang, and N. E. Bonesteel, "Violation of the entanglement area law in bosonic systems with Bose surfaces: possible application to Bose metals," Physical Review Letters, vol. 111, no. 21, Article ID 210402, 2013.

[7] M. Pouranvari and K. Yang, "Maximally entangled mode, metal-insulator transition, and violation of entanglement area law in noninteracting fermion ground states," Physical Review $B$, vol. 89, Article ID 115104, 2014.

[8] P. W. Anderson, "Absence of diffusion in certain random lattices," Physical Review, vol. 109, no. 5, pp. 1492-1505, 1958.

[9] N. Laorencie, "Scaling of entanglement entropy in the random singlet phase," Physical Review B, vol. 72, no. 14, Article ID 140408(R), 4 pages, 2005.

[10] I. Peschel, "Calculation of reduced density matrices from correlation functions," Journal of Physics A: Mathematical and General, vol. 36, no. 14, pp. L205-L208, 2003.

[11] A. MacKinnon and B. Kramer, "One-Parameter scaling of localization length and conductance in disordered systems," Physical Review Letters, vol. 47, article 1546, 1981.

[12] A. MacKinnon and B. Kramer, "The scaling theory of electrons in disordered solids: additional numerical results," Zeitschrift für Physik B: Condensed Matter, vol. 53, no. 1, pp. 1-13, 1983.

[13] D. H. Dunlap, H.-L. Wu, and P. W. Phillips, "Absence of localization in a random-dimer model," Physical Review Letters, vol. 65, no. 1, pp. 88-91, 1990.

[14] A. D. Mirlin, Y. V. Fyodorov, F.-M. Dittes, J. Quezada, and T. H. Seligman, "Transition from localized to extended eigenstates in the ensemble of power-law random banded matrices," Physical Review E-Statistical Physics, Plasmas, Fluids, and Related Interdisciplinary Topics, vol. 54, no. 4, pp. 3221-3234, 1996.

[15] R. Berkovits, "Entanglement entropy in a one-dimensional disordered interacting system: the role of localization," Physical Review Letters, vol. 108, no. 17, Article ID 176803, 5 pages, 2012.

[16] L. Pastur and V. Slavin, "Area law scaling for the entropy of disordered quasifree fermions," Physical Review Letters, vol. 113, Article ID 150404, 2014.

[17] A. C. Potter, "Boundary-law scaling of entanglement entropy in diffusive metals," http://arxiv.org/abs/1408.1094. 

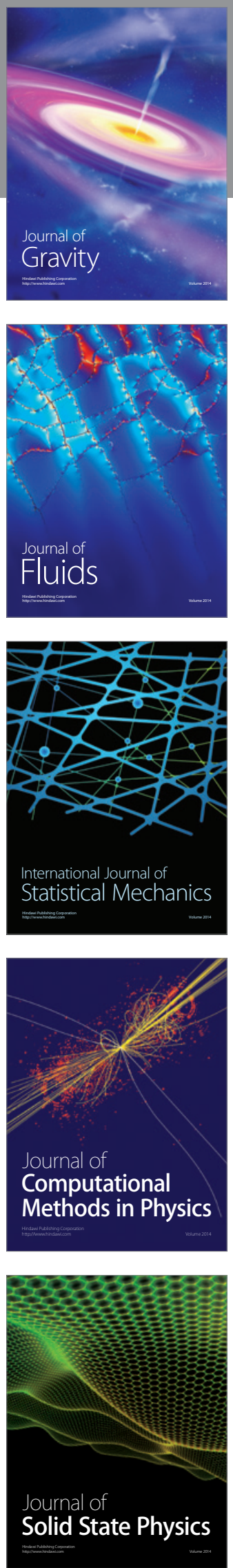

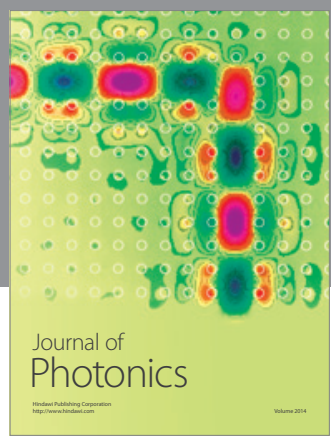

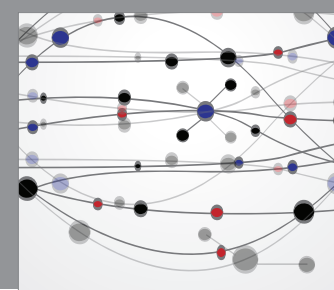

The Scientific World Journal

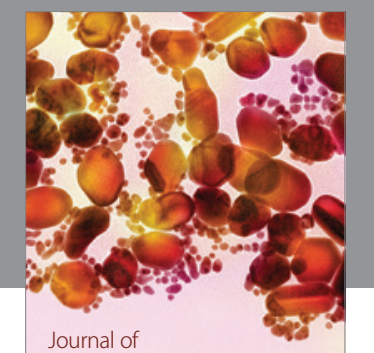

Soft Matter
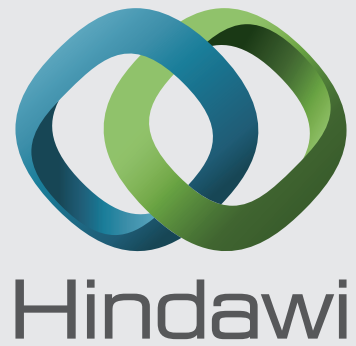

Submit your manuscripts at

http://www.hindawi.com
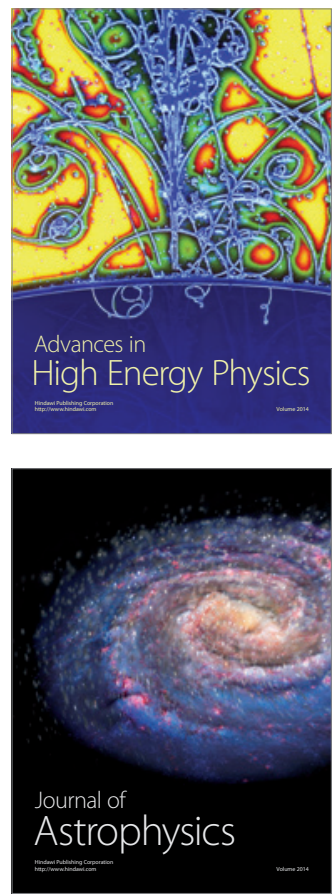
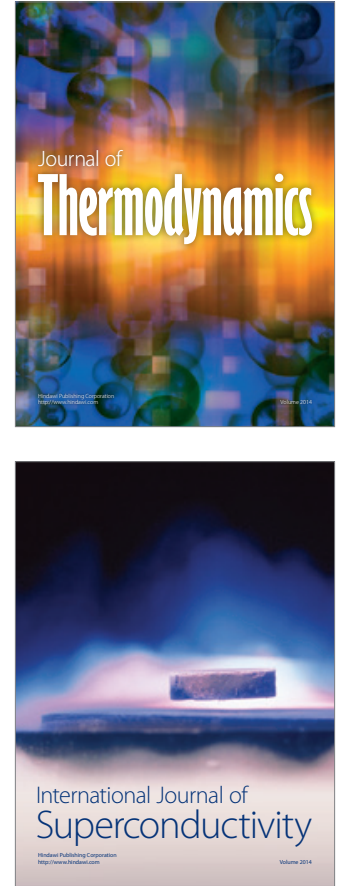
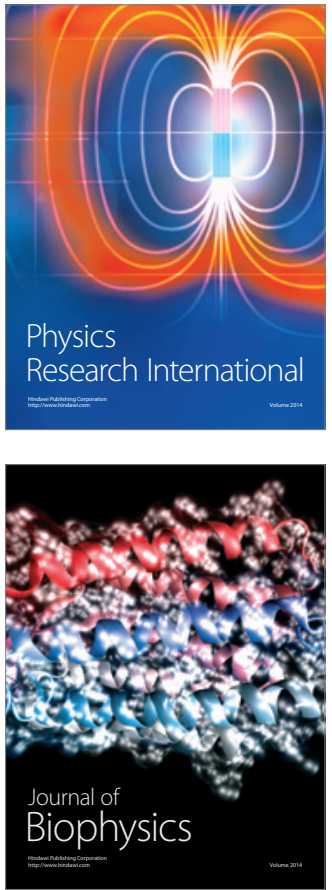
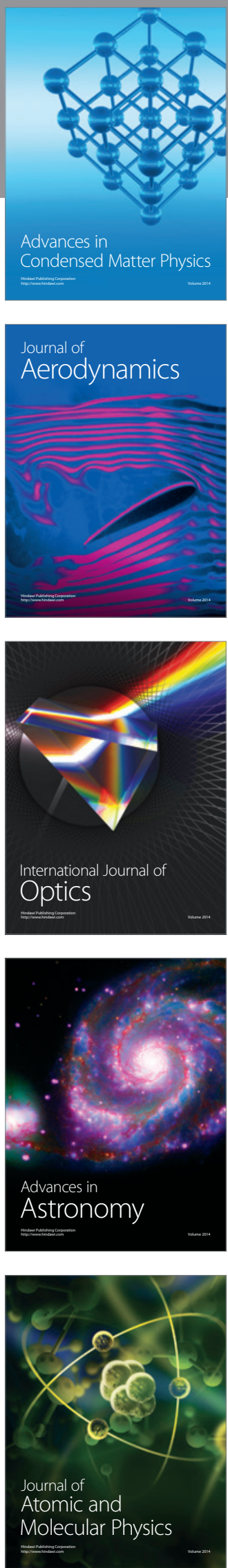